\title{
Modelo de Personalidad Positiva: su asociación al funcionamiento óptimo en trabajadores activos ${ }^{1}$
}

\author{
Guadalupe de la Iglesia ${ }^{2}$, María Laura Lupano Perugini ${ }^{3}$, \\ Alejandro Castro Solano ${ }^{4}$ \\ Consejo Nacional de Investigaciones Cientificas y Técnicas (CONICET) 2,3,4, \\ Universidad de Palermo-Argenina $a^{2,3,4}$, Universidad de Buenos Aires-Argentina ${ }^{2,3,4}$
}

El objetivo de este trabajo fue identificar si el funcionamiento óptimo de trabajadores activos —entendido como una combinación de alto bienestar y performance- podía explicarse por la presencia de rasgos positivos de la personalidad. Se trabajó con una muestra de 400 trabajadores activos argentinos de entre 18 y 66 ańos $\left(M_{E D A D}=36.50, D E=11.81,49.5 \%\right.$ varones, $50.5 \%$ mujeres). Los resultados de regresiones lineales múltiples indicaron que los rasgos positivos incrementan la varianza explicada de las variables laborales estudiadas — performance, satisfacción y bienestar - al comparárselo con un modelo de predicción que solo incluía rasgos normales. Además, en un análisis discriminante, se concluyó que de todos los rasgos — positivos y normales_ el que mejor discriminaba los grupos de alto/bajo funcionamiento óptimo era el rasgo positivo sprightliness.

Palabras clave: rasgos positivos, positive personality model, funcionamiento óptimo, psicología organizacional, personalidad.

\section{Positive Personality Model: its relation to optimal functioning in active workers}

This research aimed to analyzewhether positive traits could explain optimal functioning, the combination of high well-being and performance, of active workers. The sample comprised 400 active Argentinean workers between the ages of 18 and 66 years old $\left(M_{A G E}=36.50\right.$, $\mathrm{SD}=11.81,49.5 \%$ male, $50.5 \%$ female). Multiple lineal regressions revealed that positive traits increase the explained variance of the work variables (performance, satisfaction and

1 Investigación realizada con el apoyo del subsidio otorgado por la Universidad de Buenos Aires (UBACyT 20020150100037BA) y el Consejo Nacional de Investigaciones Científicas y Técnicas - CONICET (PIP CONICET 1122015010038).

2 Doctora en Psicología. Investigadora asistente del CONICET. Jefa de Trabajos Prácticos Universidad de Buenos Aires. Docente-investigadora del Centro de investigaciones de la Facultad de Ciencias Sociales de la Universidad de Palermo. Dirección postal: Mario Bravo 1259 (1175), Ciudad Autónoma de Buenos Aires, Argentina. Contacto: gdelaiglesia@gmail.com ORCID: 0000-0002-0420-492X

3 Doctora en Psicología. Miembro de la Carrera del Investigador del CONICET. Docenteinvestigadora Universidad de Palermo. Jefa de Trabajos Prácticos Universidad de Buenos Aires. Dirección postal: Mario Bravo 1259 (1175), Ciudad Autónoma de Buenos Aires, Argentina. Contacto: mllupano@hotmail.com ORCID: 0000-0001-6090-0762

4 Doctor en Psicología. Investigador principal del CONICET. Profesor Regular, Universidad de Buenos Aires. Dirección postal: Mario Bravo 1259 (1175), Ciudad Autónoma de Buenos Aires, Argentina. Contacto: alejandro.castrosolano@gmail.com ORCID: 0000-0002-4639-3706 
well-being) in comparison to a prediction model that only included normal traits. Also, discriminant analysis revealed that of all traits, positive and normal, the only one that better discriminated the high/low optimal functioning groups was the positive trait sprightliness. Keywords: positive traits, positive personality model, optimal functioning, organizational psychology, personality.

\section{Modelo de personalidade positiva: sua associaçáo com o ótimo funcionamento de tra- balhadores ativos}

O objetivo deste trabalho foi identificar se o funcionamento ótimo de trabalhadores ativos - entendidos como uma combinaçáo de alto bem-estar e desempenho - poderia ser explicado pela presença de traços positivos de personalidade. Trabalhamos com uma amostra de 400 trabalhadores ativos argentinos entre 18 e 66 anos de idade $(M=36.50, \mathrm{DP}=11.81$, $49.5 \%$ homens, $50.5 \%$ mulheres). Os resultados das regressóes lineares múltiplas indicam que as características positivas aumentam a variância explicada das variáveis de trabalho estudadas - desempenho, satisfaçáo e bem-estar - quando comparadas com um modelo de previsão que inclui apenas características normais. Além disso, em uma análise discriminante, concluiu-se que de todas as características - positiva e normal - a única que melhor discriminou os grupos de alta/baixa funcionalidade foi a característica positiva sprightliness. Palavras-chave: traços positivos, modelo positivo de personalidade, ótimo funcionamento, trabalhadores, psicologia organizacional, personalidade.

Modèle de personnalité positive: son association avec le fonctionnement optimal des travailleurs actifs

L'objectif de ce travail était d'identifier si le fonctionnement optimal des travailleurs actifs - compris comme une combinaison de bien-être et de performance - pouvait s'expliquer par la présence de traits de personnalité positifs. Nous avons travaillé avec un échantillon de 400 travailleurs actifs argentins âgés de 18 à 66 ans $\left(M_{\mathrm{AGE}}=36.50, E T=11.81,49.5 \%\right.$ d'hommes, $50.5 \%$ de femmes). Les résultats des régressions linéaires multiples indiquent que les traits positifs augmentent la variance expliquée des variables du travail étudiées - performance, satisfaction et bien-être - par rapport à un modèle de prédiction qui ne comprenait que des traits normaux. En outre, dans une analyse discriminante, il a été conclu que de tous les traits - positifs et normaux - le seul qui a le mieux discriminé les groupes fonctionnels haut / bas était le trait positif vivacité.

Mots clés: traits positifs, modèle de personnalité positive, fonctionnement optimal, travailleurs, psychologie organisationnelle, personnalité. 
En el campo de la psicología laboral y organizacional, cobra especial relevancia el estudio de factores de personalidad que pueden estar relacionados con el funcionamiento óptimo de los empleados. En este sentido, un creciente número de investigaciones sugieren que la personalidad de los trabajadores se vincula con la obtención de óptimos resultados, como el desempeño laboral (Barrick, Mount \& Judge, 2001), los niveles de rotación (Zimmerman, 2008), el ausentismo (Salgado, 2002) y la satisfacción laboral (Jugde, Heller \& Mount, 2002).

En el presente estudio, se buscará analizar el poder predictivo de determinados rasgos de la personalidad sobre el funcionamiento óptimo de trabajadores activos, entendido como la combinación de altos niveles de performance y bienestar. Un número importante de estudios han analizado la relación entre rasgos de personalidad y el rendimiento o performance. En virtud de que son muchas las investigaciones que han abordado este tópico pueden consultarse diversos metaanálisis que sintetizan los hallazgos. Si se consideran los estudios que se han basado en el Modelo de los Cinco Grandes o Five Factor Model (FFM) (Costa \& McCrae, 1985), puede mencionarse que en general el rasgo que mayor incidencia presenta sobre los niveles de performance es el de responsabilidad (Barrick \& Mount, 1991; Salgado, 1997). En relación al resto de los rasgos del modelo, se ha hallado que el poder de predicción varía de acuerdo al tipo de ocupación. Por ejemplo, el rasgo extraversión es un buen predictor para todos aquellos grupos ocupacionales que impliquen interacción social —e.g. ventas (e.g. Anderson \& Viswesvaran, 1998; Barrick \& Mount, 1991; Salgado 1997; Tett, Jackson \& Rothstein, 1991) —. La mayor parte de los estudios concluyen que existen variables moderadoras que intervienen en el efecto sobre la performance. En este sentido, Christiansen y Tett (2008) sostienen que, considerando a los rasgos de forma individual, los valores de correlación con performance son bajos, oscilando entre 0 y .30. 
Además, varios estudios han analizado la relación de determinados rasgos de personalidad con la satisfacción laboral. Dentro de los factores del FFM se pueden vislumbrar algunos antecedentes empíricos que dan cuenta de dicha relación. Heller, Ferris, Brown \& Watson (2009), por ejemplo, hallaron que el neuroticismo predice la disminución de la satisfacción laboral; y la extraversión, la apertura a la experiencia, la amabilidad y la responsabilidad predicen su incremento. Esto ocurre tanto cuando se miden los rasgos de personalidad de manera general como cuando se los mide contextualizados en el trabajo. Adicionalmente, los rasgos de responsabilidad, extraversión, amabilidad y estabilidad emocional se asocian positivamente a la satisfacción laboral y negativamente a la frustración en el trabajo, la intención de renuncia y el ausentismo (Bowling \& Burns, 2010). Por otra parte, Mount, Ilies y Johnson (2006), indicaron que los cinco grandes rasgos de la personalidad están asociados negativamente con conductas laborales contraproducentes. En relación con lo antedicho, un metaanálisis en el que se estudió la relación entre el FFM y el burnout laboral llegó a concluir que la extraversión, amabilidad y responsabilidad se encuentran asociados negativamente a la presencia del burnout a excepción del neuroticismo que se asocia de forma positiva y fuerte con la presencia del síndrome (Swider \& Zimmerman, 2010).

Por último, en cuanto a la relación con el bienestar, si se toma la conceptualización de Keyes (2002), que entiende al bienestar en sus tres dimensiones (social, personal y emocional), algunos estudios han mostrado que los rasgos normales funcionan como predictores del bienestar (Strickhouser, Zell \& Krizan, 2017), y que, específicamente, la extraversión y la agradabilidad son los que mayor relación positiva tienen con esta noción de bienestar. En contraposición, el neuroticismo suele estar asociado de forma negativa con el bienestar (DeNeve \& Harris, 1998; Lamers, Westerhof, Kovács \& Bohlmeijer, 2012), incluso en países no occidentales (Zhai, Willis, O’Shea, Zhai \& Yang, 2013). 


\section{Positive Personality Model: los rasgos positivos de la personalidad}

Los antecedentes mencionados se basan en el conocido modelo FFM de rasgos normales de personalidad. El presente estudio trata de incorporar un novedoso modelo que propone la evaluación de los rasgos positivos de la personalidad. La importancia de este modelo es que ha demostrado un poder predictivo mayor sobre los niveles de bienestar en comparación con el FFM.

El Positive Personality Model (PPM) -Modelo de Personalidad Positiva- constituye un modelo de sanidad integrado al modelo patológico propuesto en la sección III del DSM-5 (De la Iglesia \& Castro Solano, 2018). La novedosa propuesta de evaluación dimensional de los trastornos de la personalidad (TP) presentada en la sección III del Manual Diagnóstico y Estadístico de los Trastornos Mentales (DSM-5; American Psychiatric Association, 2013), trajo a colación la ya conocida noción de continuidad sindrómica entre la personalidad patológica y normal (e.g., Leary, 1957; Millon, 1996; Millon \& Everly, 1994; Offer \& Sabshin, 1991; Strack \& Lorr, 1994).

La disponibilidad de un marco conceptual que integre clasificaciones patológicas y normales ha llegado a generar un aumento de interés en la comunidad científica y del ámbito aplicado. En este sentido se constituyó en la mencionada propuesta del DSM-5 en donde, de la mano de Krueger et al. (2013), se presentó el Personality Inventory for DSM-5 (PID-5). Este instrumento mide cinco rasgos patológicos desde una perspectiva empírica y dimensional. Los cinco rasgos resultan ser versiones patológicas de los incluidos en el Modelo de los Cinco Grandes. En consecuencia, una clasificación de rasgos normales de la personalidad quedó integrada a una clasificación de rasgos patológicos de la personalidad en un mismo marco conceptual, hecho que anteriormente había sido rechazado (Krueger, Derringer, Markon, Watson \& Skodol, 2013; Millon, 1996).

Los rasgos patológicos evaluados por el PID-5 son: afecto negativo, desapego, antagonismo, desinhibición y psicoticismo. A pesar de ser presentados como versiones patológicas de los rasgos del FFM —estabilidad emocional, extraversión, agradabilidad, responsabilidad 
y apertura a la experiencia-, en el DSM-5 no se incluyó más información al respecto (American Psychiatric Association, 2013). Sumado a ello, a pesar de estar integrado al FFM, el modelo de Krueger et al. (2013), no deja de estar únicamente centrado en la descripción y valoración de patologías psicológicas sin referir a la promoción y valoración de aspectos saludables. Sin embargo, actualmente se acepta que la ausencia de síntomas psicopatológicos no es garantía ni equivale al funcionamiento óptimo de los sujetos o a una buena calidad de vida (Seligman \& Csikzentmihalyi, 2014). En consecuencia, en el terreno de los rasgos de la personalidad, es necesario contar con un modelo que valore sus aspectos positivos y que, idealmente, esté integrado a la propuesta vigente por el DSM-5.

El modelo aquí incluido (PPM), constituye un intento de integración con la clasificación de patología de la personalidad propuesta en la sección III del DSM-5. El PPM presenta, como novedad, la incorporación de versiones positivas de los cinco rasgos patológicos propuestos por Krueger et al. (2013), estos son: serenidad, humanidad, integridad, moderación y sprightliness. Estos cinco rasgos positivos se ubican en el continuum de salud-enfermedad previamente mencionado constituyendo un polo adicional ubicado más allá de la normalidad: el polo positivo. Específicamente, el rasgo serenidad es el opuesto positivo de afecto negativo y neuroticismo, y se caracteriza por un estado de paz y calma. El rasgo humanidad es la versión positiva de desapego y de extraversión e implica una alta sensibilidad al contexto. El rasgo integridad resulta ser el polo opuesto de antagonismo y la versión positiva de afabilidad, se caracteriza por la confianza y humildad. El rasgo moderación -opuesto a desinhibición y versión positiva de responsabilidadimplica cautela y reflexión. Por último, sprightliness — versión positiva de apertura a la experiencia y el opuesto de psicoticismo - se relaciona con la autoconfianza, tener metas claras, ser activo y sentirse satisfecho.

Esta propuesta fue respaldada por los análisis realizados en su validación en los que se halló que los rasgos positivos están asociados parcial y positivamente a los rasgos del FFM, y están asociados negativamente a los rasgos patológicos del PID-5. Además, al comparárselos 
con los rasgos normales, los rasgos positivos presentaron evidencias de validez incremental funcionando como mejores predictores de la salud mental positiva en población general. El fin último del PPM, radica en funcionar como un manual de sanidades actualizado e integrado a las nociones más nuevas en el diagnóstico de la personalidad.

\section{Rasgos de personalidad positivos y funcionamiento óptimo en trabajadores activos}

En relación con los rasgos positivos, no existen antecedentes desde el PPM debido a su reciente postulación. Sin embargo, pueden realizarse ciertos paralelismos con el modelo de virtudes y fortalezas denominado VIA — Values in Action - (Peterson \& Seligman, 2004). En términos generales, la presencia de fortalezas del carácter se asocia a una mejor performance en el trabajo. Dubreuil et al. (2016), por ejemplo, encontraron que luego de una intervención que promovía el incremento de las fortalezas del carácter, se incrementaba la performance laboral. Harzer y Ruch (2014), hallaron que las fortalezas vitalidad y perseverancia - equiparables al rasgo sprightliness en el PPM—, amabilidad y perdón —equiparables a humanidad-, autorregulación —equiparable a serenidad_, prudencia —equiparable moderación-, y honestidad y justicia — equiparables a integridad—, se encontraban asociadas a una mejor performance laboral. Resultados similares fueron reportados por Moradi, Nima, Ricciardi, Archer y Garcia (2014), quienes hallaron una asociación positiva entre performance y las fortalezas autorregulación, prudencia, humildad y modestia. Sin embargo, encontraron asociaciones negativas con la fortaleza humanidad entre otras. Similarmente, Littman-Ovadia y Lavy (2016) hallaron que las fortalezas perseverancia, honestidad, prudencia y amabilidad estaban asociadas positivamente a la productividad y negativamente a los comportamientos contra-productivos en el trabajo. Estas fortalezas pueden equipararse a los rasgos positivos sprightliness, integridad, moderación y humanidad, respectivamente. Por otra parte, la fortaleza autorregulación, también equiparable en el PPM al rasgo serenidad, estaba asociada a la baja en los comportamientos contraproductivos. 
En relación con los índices de satisfacción laboral, LittmanOvadia, Lavy y Boiman-Meshita (2016) encontraron en un grupo de trabajadores activos que estaba asociada a las fortalezas esperanza, amor, gratitud, curiosidad y vitalidad. En otro estudio, Lavy y LittmanOvadia (2016) reportaron que el uso de las fortalezas de carácter en el trabajo estaba asociado a la satisfacción laboral y la productividad.

Por último, en cuanto al bienestar de los empleados, Harzer, Mubashar y Dubreuil (2017), hallaron que estaba asociado a distintas fortalezas del carácter como vitalidad, trabajo en equipo, esperanza, amor, gratitud, liderazgo y perseverancia. Algunas de ellas son equiparables al rasgo sprightliness y humanidad del PPM.

En función de lo expuesto se delinearon los siguientes objetivos: (1) Analizar la validez incremental de los rasgos positivos del PPM por sobre los rasgos normales del FFM en términos de predicción de la performance, la satisfacción laboral y el bienestar; (2) Identificar grupos diferenciados de acuerdo al grado de funcionamiento óptimo - combinación de performance y bienestar-; y (3) Estudiar mediante un análisis discriminante qué variables del PPM contribuyen más a la separación de los grupos identificados en el objetivo anterior.

\section{Método}

\section{Participantes}

La muestra estaba constituida por 400 adultos argentinos que eran trabajadores activos al momento de ser evaluados. La edad promedio fue de $36.50(D E=11.81$, Min $=18$, Máx = 66). El 49.5\% $(n=198)$ eran varones y el $50.5 \%(n=202)$ eran mujeres. En cuanto a su lugar de residencia, el 42.75\% $(n=171)$ residía en la Ciudad Autónoma de Buenos Aires, el 24\% $(n=96)$ en el Gran Buenos Aires, y el 33.25\% $(n=133)$ en alguna provincia del país. El $28.5 \%(n=114)$ dijo estar casado, el 27.3\% $(n=109)$ ser soltero, el 19.5\% $(n=78)$ estar de novio, el $18.3 \%(n=73)$ estar conviviendo con su pareja, el $5.3 \%(n=21)$ estar divorciado y el $1.3 \%(n=5)$ ser viudo. En cuanto a su educación, 
el $9.75 \%(n=39)$ dijo no haber completado la secundaria, el $17.5 \%$ $(n=69)$ indicó haber finalizado la secundaria, el 27.5\% $(n=110)$ estar cursando estudios universitarios o terciarios, el 39.25\% $(n=157)$ haber completado estudios universitarios o terciarios, el $2 \%(n=8)$ estar cursando un posgrado y el $4.25 \%(n=17)$ haber finalizado un posgrado. El $67.8 \%(n=271)$ dijo pertenecer a la clase media, el $18.5 \%(n=74)$ a la clase media-baja, el $12.3 \%(n=49)$ a la clase media-alta, el $1 \%$ $(n=4)$ a la clase baja y el $0.5 \%(n=2)$ a la clase alta.

En cuanto a variables organizacionales, el 58.8\% $(n=233) \mathrm{de}$ los empleados pertenecía a empresas privadas mientras que el $41.8 \%$ $(n=167)$ se desempeñaba en empresas públicas. En cuanto al tamaño de la empresa, el 39.3\% $(n=157)$ pertenecía a grandes empresas, el $32.8 \%(n=131)$ a medianas y el $28 \%$ a pequeńas $(n=112)$. La mayor parte de los participantes tenía personal a cargo $(74.5 \%, n=298)$, en tanto que el $25.5 \%$ ocupaba una posición de subordinado.

\section{Medición}

Para la medición de rasgos positivos de la personalidad se utilizó el Inventario de Rasgos Positivos - 5 (IRP-5; De la Iglesia \& Castro Solano, 2018), que brinda una medida de dichos rasgos mediante 60 ítems y que se responden mediante una escala Likert de seis posiciones que van de 0 (completamente falso) a 6 (completamente verdadero). El instrumento fue diseñado como una versión inversa del Personality Inventory for DSM-5 (PID-5) - Adult (Krueger et al., 2013), que mide cinco rasgos patológicos de la personalidad de acuerdo a la propuesta de la sección III del Manual Diagnóstico y Estadístico de los Trastornos Mentales en su quinta edición (DSM-5; American Psychiatric Association, 2013). Los cinco rasgos positivos evaluados por el IRP-5 son las siguientes: serenidad (13 ítems) una medida de tranquilidad casi imperturbable y de excelente manejo de las emociones negativas propias y ajenas; humanidad (8 ítems) refiere a quienes son sumamente sensibles a su entorno y no temen mostrar su sensibilidad, además de que el malestar ajeno los moviliza al punto de actuar con el fin de terminarlo; el rasgo integridad (13 ítems) se refiere a quienes son confiables y sinceros, que no dudan 
en admitir sus errores y son humildes esperando un trato igualitario a los demás; moderación (9 ítems) es un rasgo que contempla la cautela y la previsión y evaluación de posibles riesgos ante la toma de decisiones, y finalmente el rasgo de sprightliness (17 ítems) contiene ítems que valoran la capacidad de saber cuáles son los propios objetivos, logrando una alta concentración al llevarlos a cabo y vivenciando un gran bienestar al hacerlo. Los estudios psicométricos realizados sobre la medida indicaron excelentes evidencias de validez de constructo para la estructura de cinco factores mediante los resultados obtenidos en análisis factoriales exploratorios y confirmatorios. Además, los estudios de validez convergente — con una medida del FFM - y discriminante - con el PID-5- brindaron evidencias suficientes para ubicar a los cinco rasgos del IRP-5 como representantes del polo positivo del continuo salud-enfermedad de los rasgos de personalidad. La consistencia interna del instrumento del estudio original era excelente y en la muestra aquí utilizada los alfas de Cronbach obtenidos fueron serenidad $=.87$, humanidad $=.81$, integridad $=.83$, moderación $=.79$ y sprightliness $=.89$.

El bienestar fue evaluado mediante el Mental Health ContinuumShort Form (MHC-SF) (Keyes, 2005; adaptación argentina, Lupano Perugini, de la Iglesia, Castro Solano \& Keyes, 2017). Se trata de un instrumento de 14 ítems con una escala Likert de 5 puntos, que va de 0 (nunca) a 5 (todos los días), destinada a la medición del bienestar - $\mathrm{O}$ salud mental positiva - en sus aspectos emocionales (3 ítems), psicológico (6 ítems) y sociales (5 ítems). El MHC-SF ha demostrado tener una muy buena consistencia interna $(>.70)$ y validez discriminante en adultos en varios estudios internacionales. En la adaptación argentina ha confirmado la estructura tridimensional de la escala y una buena validez convergente. Para este estudio se utiliza únicamente la puntuación total como una medida bienestar. El alfa obtenido para la escala total es de .89.

Además, se diseñaron una serie de encuestas para la evaluación de algunas de las variables consideradas en el estudio: En primer lugar, se diseñó una Encuesta organizacional que consultaba a los participantes sobre datos de la organización en la que trabajan y el puesto que ocupan (tamaño, tipo, rubro, área en la que trabaja, puesto y personal a cargo). 
Luego, con la finalidad de medir el nivel de satisfacción laboral se construyó una Encuesta de Satisfacción Laboral que consta de seis ítems con opción Likert de respuesta que va de 1 (totalmente insatisfecho) a 7 (totalmente satisfecho) y que evalúan cuán satisfecha se autopercibe la persona en cuanto a su trabajo en general y diferentes aspectos de este (sueldo, jefes, compañeros, lugar y carrera). Un ejemplo de ítem es «¿Cuán satisfecho estoy con el sueldo que recibo?». Para la elección de las áreas a evaluar (p. ej., sueldo, jefes, compañeros) se tomaron en cuenta aspectos analizados en otros instrumentos previos (p. ej., Balzer et al., 1997). Se calculó alfa de Cronbach para la escala total obteniéndose un valor aceptable de .79

Por último, para la evaluación del desempeño se diseñó una Encuesta sobre Desempeño-performance Organizacional e Individual. Para la construcción de esta encuesta, se tomaron como indicadores de desempeño los propuestos por Cameron, Brigth, y Caza (2004), en sus investigaciones sobre variables positivas y performance (i.e., eficiencia, innovación, crecimiento, calidad, retención de empleados y clientes, satisfacción, adaptación). La primera parte de la encuesta, consta de diez ítems con opción Likert de respuesta -1 (Poco) a 6 (Mucho)- y está orientada a evaluar la performance organizacional de acuerdo con la percepción de los empleados. Un ejemplo de ítem es «¿En qué medida cree que la organización cumplió eficientemente - haciendo un buen uso de los recursos- con los objetivos propuestos?». El coeficiente alfa obtenido para este apartado fue de .91. La segunda sección, destinada a la evaluación de la performance individual, consta de seis ítems con la misma opción de respuesta que el apartado anterior $(1=$ Poco y $6=M u c h o)$. Un ejemplo de ítem es: «¿En qué medida cree que los resultados obtenidos fueron de calidad?». El coeficiente alfa obtenido para esta segunda parte fue de .85 .

\section{Procedimiento}

Los datos fueron recolectados por alumnos que se encontraban realizando una práctica de investigación en una universidad privada de la ciudad de Buenos Aires. Los participantes fueron voluntarios 
y no recibieron retribución alguna por su colaboración. Además, el cuadernillo que contenía las encuestas presentaba en su portada una introducción en la que se solicitaba el consentimiento del participante, se aseguraba el anonimato de los datos y su uso exclusivo para investigación. La recolección y carga de datos fue supervisada por un docente investigador.

\section{Análisis de datos}

Para la carga y análisis de los datos se utilizó el software SPPS 18.0. En el caso del análisis de validez incremental se utilizó un análisis de regresión jerárquica múltiple. Para la identificación de grupos de acuerdo al funcionamiento óptimo un análisis de conglomerados jerárquico utilizando el método Ward y la distancia euclídea al cuadrado. Finalmente, para el análisis de diferenciación de los grupos de funcionamiento óptimo de acuerdo a los rasgos positivos se calculó un análisis discriminante descriptivo.

\section{Resultados}

\section{Validez incremental de los rasgos positivos}

Se calculó un análisis de regresión jerárquica múltiple para poder estudiar si los rasgos positivos incrementaban la predicción de la performance, satisfacción laboral y bienestar de los trabajadores, más allá de la varianza explicada por los rasgos normales del FFM. Primero se introdujeron los cinco factores del FFM como predictores del primer bloque y, luego, se introdujeron los rasgos positivos en el segundo bloque. En todos los casos los modelos fueron estadísticamente significativos $(p<.01)$ y los rasgos positivos incrementaban significativamente el cambio en $R^{2}$ (ver Tabla 1 ).

En el caso de la performance el $R^{2}$ del Bloque 1 fue de .133 $[F(5,394)=13.25, p<.001]$, y el único rasgos que resultó ser un predictor estadísticamente significativo fue responsabilidad $(\beta=.270, p<$ $.001)$. En el Bloque 2 el $R^{2}$ pasó a ser $.198[F(10,389)=10.87, p<.001]$ 
y este cambio fue estadísticamente significativo $(p<.001)$ e implicó un aumento del $6.5 \%$ de la varianza explicada. El rasgo responsabilidad $(\beta=.129, p=.029)$ mantuvo su significancia estadística como predictor de la performance, y, dentro de los rasgos positivos, el rasgo sprightliness $(\beta=.263, p<.001)$ resultó ser un predictor estadísticamente significativo.

\section{Tabla 1}

Regresión jerárquica múltiple: predicción de performance, satisfacción y salud mental positiva $(n=400)$

\begin{tabular}{|c|c|c|c|c|c|c|}
\hline & \multicolumn{2}{|c|}{ Performance } & \multicolumn{2}{|c|}{$\begin{array}{c}\text { Satisfacción del } \\
\text { empleado } \\
\end{array}$} & \multicolumn{2}{|c|}{ Bienestar } \\
\hline & $R^{2}$ & $\beta$ & $R^{2}$ & $\beta$ & $R^{2}$ & $\beta$ \\
\hline Bloque 1 & $.133^{* * *}$ & & $.077^{* * *}$ & & $.173^{* * *}$ & \\
\hline Neuroticismo & & -.047 & & -.053 & & $-.205^{* * *}$ \\
\hline Extraversión & & .098 & & $.109^{*}$ & & $.197^{* * *}$ \\
\hline Agradabilidad & & .045 & & .105 & & .026 \\
\hline Responsabilidad & & $.270^{* * *}$ & & $.166^{*}$ & & $.157^{* *}$ \\
\hline Apertura a la experiencia & & .077 & & -.090 & & .055 \\
\hline Bloque 2 & $.198^{* *+*}$ & & $.103^{* * *}$ & & $.329^{* *+*}$ & \\
\hline Neuroticismo & & -1.011 & & .031 & & -.102 \\
\hline Extraversión & & .040 & & .075 & & $.140^{* *}$ \\
\hline Agradabilidad & & .005 & & .122 & & .005 \\
\hline Responsabilidad & & $.127^{*}$ & & .078 & & -.026 \\
\hline Apertura a la experiencia & & .032 & & $-.105^{*}$ & & -.003 \\
\hline Sprightliness & & $.263^{* *+}$ & & $.213^{* *}$ & & $.468^{* * *+}$ \\
\hline Integridad & & .105 & & .064 & & $-.161^{* *}$ \\
\hline Serenidad & & -.093 & & .020 & & .115 \\
\hline Moderación & & .044 & & -.008 & & .019 \\
\hline Humanidad & & .042 & & -.113 & & $.111^{*}$ \\
\hline
\end{tabular}


Cuando el criterio a predecirse fue la satisfacción del empleado, el $R^{2}$ del Bloque 1 fue de $.077[F(5,394)=7.63, p<.001]$. Los rasgos normales que resultaron ser predictores estadísticamente significativos dentro de este bloque fueron: extraversión $(\beta=.109, p=.046)$ y responsabilidad $(\beta=.166, p=.003)$. El $R^{2}$ del Bloque 2 fue .103 $[F(5,389)=6.09, p<.001]$ dándose un incremento del $2.6 \%$ de la varianza explicada y siendo este cambio estadísticamente significativo $(p<.001)$. Al introducir los rasgos positivos en el modelo de predicción, los rasgos de extraversión y responsabilidad perdieron su significancia estadística. El rasgo apertura a la experiencia $(\beta=-.105, p=.048)$, en cambio, es un predictor estadísticamente significativo en este modelo junto con el rasgo positivo sprightliness $(\beta=.213, p=.003)$.

Por último, con relación al bienestar, el $R^{2}$ del Bloque 1 fue .173 $[F(5,394)=17.74, p<.001]$. Los predictores estadísticamente significativos fueron neuroticismo $(\beta=-.205, p<.001)$, extraversión $(\beta=.197$, $p<.001)$ y responsabilidad $(\beta=.157, p=.003)$. El cambio en el $R^{2}$ al calcularse el Bloque 2 fue estadísticamente significativo $(p<.001)$ y el $R^{2}$ fue de $.329[F(10,389)=20.59, p<.001]$, alcanzando en este caso un incremento del $15.6 \%$ de la varianza explicada. En este bloque, el único rasgo normal que resultó ser un predictor estadísticamente significativo fue extraversión $(\beta=.140, p=.005)$. Dentro de los rasgos positivos, resultaron ser predictores estadísticamente significativos sprightliness $(\beta=.468, p<.001)$, integridad $(\beta=-.161, p=.005) \mathrm{y}$ humanidad $(\beta=.111, p=.038)$.

\section{Diferenciación de grupos de acuerdo con el funcionamiento óptimo}

Se buscó identificar conglomerados de acuerdo con el nivel de funcionamiento óptimo. Para ello se calculó un análisis de conglomerados jerárquico utilizando el método Ward y la distancia euclídea al cuadrado. Esto se hizo para determinar el número adecuado de clusters que maximizaba la diferencia entre los grupos y minimizaba las diferencias intragrupales. El historial del coeficiente de conglomeración y el dendograma indicaron que una solución de dos conglomerados era la más adecuada. 
A continuación, se solicitaron dos clusters en el análisis de conglomerados de $\mathrm{k}$ medias. Los ANOVAs indicaron que existían diferencias estadísticamente significativas en la performance y salud mental positiva de acuerdo con la pertenencia al cluster $(p<.01)$. En la tabla 2 se pueden visualizar las medias para performance y salud mental positiva según el cluster de pertenencia. Puede observarse que el cluster 1 puntuó bajo performance y en bienestar por lo que podría denominárselo «bajo funcionamiento óptimo", y el cluster 2 puntuó alto en performance y bienestar por lo que podría denominárselo «alto funcionamiento óptimo». Luego, los resultados de un MANOVA indicaron que existían diferencias estadísticamente significativas en los cinco rasgos positivos de acuerdo con la pertenencia al cluster $(\lambda$ de Wilks $(5,394)=.77$, $p<.01, \eta^{2}=.22$ ). Las pruebas a posteriori de Bonferroni indicaron que los sujetos pertenecientes al cluster "alto funcionamiento positivo" presentaban en mayor medida todos los rasgos positivos $(p<.05)$.

\section{Tabla 2}

Clusters de funcionamiento óptimo

\begin{tabular}{lccccc}
\hline & $\begin{array}{c}\text { Cluster } 1 \\
(n=185)\end{array}$ & $\begin{array}{c}\text { Cluster } 2 \\
(n=215)\end{array}$ & $F$ & $p$ & $\eta^{2}$ \\
\cline { 2 - 6 } & -.67 & .58 & 249.46 & $<.001$ & .385 \\
$\begin{array}{l}\text { Performance } \\
\text { (puntuación tipificada) }\end{array}$ & -.76 & .65 & 387.44 & $<.001$ & .493 \\
$\begin{array}{l}\text { Bienestar } \\
\text { (puntuación tipificada) }\end{array}$ & 3.50 & 4.08 & 15.62 & $<.001$ & .038 \\
$\begin{array}{l}\text { Sprightliness } \\
\text { Integridad }\end{array}$ & 4.00 & 4.29 & 16.80 & $<.001$ & .040 \\
Serenidad & 3.16 & 3.47 & 5.00 & .026 & .012 \\
Moderación & 3.47 & 3.62 & 30.14 & $<.001$ & .070 \\
Humanidad & 3.42 & 3.74 & 109.86 & $<.001$ & .216 \\
\hline
\end{tabular}




\section{Discriminación de grupos de acuerdo con los rasgos positivos}

Por último, se buscó identificar qué rasgos positivos contribuían más a maximizar la separación entre el grupo de alto funcionamiento óptimo y el de bajo funcionamiento óptimo. Para ello se calculó un análisis discriminante descriptivo (Tabachnick \& Fidell, 2007). La función discriminante presentó una correlación canónica de .47 ( $\lambda$ de Wilks $\left.=.78, \chi^{2}=100.48, \mathrm{gl}=5, p<.001\right)$.

La selección de las variables que más contribuían a maximizar la separación entre los grupos de funcionamiento óptimo estuvo fundamentada en el análisis de su correlación con la función discriminante y la verificación de que la $F$ ajustada de acuerdo con las otras variables fuera estadísticamente significativa (Tabachnick \& Fidell, 2007). La única variable que cumplía con estos criterios de selección fue el rasgo positivo sprightliness, $r=.96, F(1,398)=109.86, p<.001$. Es decir, que el rasgo que más discrimina a los grupos de alto y bajo funcionamiento óptimo es el sprightliness que implica una buena capacidad de concentración, foco en el objetivo y en la tarea, sumado a sensación de energía y satisfacción consigo mismo.

\section{Discusión}

El objetivo de este trabajo radicó en el análisis de los rasgos positivos propuestos por el PPM (De la Iglesia \& Castro Solano, 2018) en una muestra de trabajadores activos. Se buscó identificar si los rasgos positivos aumentaban la varianza explicada de manera significativa en la predicción de funcionamiento óptimo en esta población que engloba las variables performance del empleado y bienestar.

En relación con la performance, los rasgos positivos presentaron validez incremental explicando significativamente mayor varianza que el modelo que solo incluía a los rasgos normales de personalidad. $\mathrm{El}$ análisis de los predictores individuales incluidos en el modelo del Bloque 2 indicó que los rasgos responsabilidad y sprightliness eran predictores estadísticamente significativos de la performance del empleado. 
Este resultado se condice con los antecedentes mencionados (Barrick \& Mount, 1991; Salgado, 1997) y denotan que no son únicamente necesarias las características implicadas en el rasgo responsabilidad dadas por la organización, la eficiencia, la disciplina que favorecen el logro de la tarea, sino que también será necesaria la presencia de las características positivas implicadas en el rasgo sprightliness que incluyen el foco en el objetivo, el bienestar, la concentración y la vitalidad. Sería interesante tener en cuenta estos hallazgos en el contexto aplicado (empresas y organizaciones laborales) a fin de incluir el análisis de rasgos positivos a la hora de analizar los perfiles de potenciales candidatos, ya que ha quedado demostrado que estos predicen un óptimo desempeño más allá de los rasgos normales de personalidad que, como se detalló en este estudio, son los que comúnmente se evalúan.

En cuanto a la satisfacción laboral, los resultados también demostraron que los rasgos positivos aportaban significativamente a la varianza explicada de la variable criterio. Los rasgos responsabilidad y extraversión que resultaban ser predictores estadísticamente significativos en el Bloque 1, perdieron su significancia estadística en el Bloque 2 en donde los predictores estadísticamente significativos resultaron ser apertura a la experiencia y sprightliness. Nuevamente, sprightliness emerge como uno de los rasgos más importantes en cuanto a la predicción del criterio. En comparación con los antecedentes, puede decirse que los resultados del Bloque 1 condicen con el estado del arte (p. ej., Bowling $\&$ Burns, 2010; Heller et al., 2009). Sin embargo, los resultados del Bloque 2 los contradicen, dando mayor relevancia a los rasgos positivos y al rasgo apertura a la experiencia. Entre los resultados de LittmanOvadia et al., (2016) cabe mencionarse que la fortaleza vitalidad estaba asociada a una mayor satisfacción laboral. Esto puede ubicarse como un precedente en cuanto a lo hallado con el rasgo sprightliness. Además, debe tenerse en cuenta que el rasgo sprightliness es la versión positiva de apertura a la experiencia en el PPM, por lo que cobra sentido que ambos rasgos sean buenos predictores de la satisfacción laboral y que en el Bloque 2 el rasgo apertura a la experiencia tenga un coeficiente de predicción negativo. Este resultado denota que ambos rasgos no 
evalúan un mismo constructo y que sprightliness es una versión distinta y positiva de apertura a la experiencia.

Por último, los rasgos positivos también incrementaban significativamente la predicción de bienestar. Esto se condice con lo hallado por De la Iglesia y Castro Solano (2018), y aporta a la evidencia a favor de la postulación de los rasgos positivos son mejores predictores del bienestar que los rasgos normales. De acuerdo con los resultados, nuevamente sprightliness aparece como el predictor más fuerte. El rasgo integridad resultó ser un predictor negativo del bienestar, lo que daría cuenta que, en la muestra de trabajadores aquí analizada, quienes se autoperciben como confiables, humildes, sinceros y capaces de admitir sus errores son menos proclives a experimentar bienestar tanto a nivel emocional, como personal y social. Además, el rasgo humanidad resultó ser un predictor significativo del bienestar. Quienes tienen una alta sensibilidad al contexto en el que están con propensión a detectar la necesidad de ayuda ajena y actuar respondiendo a esa necesidad, tienen mayores niveles de bienestar.

En relación a los rasgos normales de personalidad, el rasgo de extraversión es el que se mantiene como predictor en ambos bloques, siendo coincidente con los hallazgos previos (Lamers et al., 2012). Una predisposición a la actividad y contacto social contribuye a experimentar bienestar desde la conceptualización aquí escogida (Keyes, 2002).

Pasando ahora a la clasificación de los grupos de funcionamiento óptimo alto/bajo, el análisis discriminante informó que la variable que mejor discriminaba a los grupos era el rasgo positivo sprightliness. Esto se condice con los antecedentes que indican una mejor predicción de funcionamiento óptimo por parte de las características positivas por sobre los rasgos normales (Drozd, Mork, Nielsen, Raeder, Bjorkli, 2014; Duan et al., 2012; Elston \& Boniwell, 2011; Gander, Proyer, Ruch \& Wyss, 2012; Meyers \& Van Woerkom, 2014; Mongrain \& AnselmoMatthews, 2012; Wood, Linley, Matlby, Kashdan \& Hurling, 2011; Young, Kashdan \& Macatee, 2014). Además, da aún mayor relevancia al rasgo positivo sprightliness, que a lo largo de estos resultados fue el más asociado a los criterios aquí estudiados. 
Una limitación del estudio presentado se relaciona con que los rasgos de personalidad fueron evaluados de modo general, y no se consideraron diferencias de acuerdo con tipos de ocupación y sus respectivas tareas. Los antecedentes consultados coinciden en sostener que es necesario analizar si determinadas ocupaciones pueden tener mayor relación con determinados rasgos de personalidad (p. ej. Anderson \& Viswesvaran, 1998; Barrick \& Mount, 1991; Salgado 1997; Tett et al., 1991). Por ejemplo, el postulado central de la Trait Activation Theory - Teoría de Activación del Rasgo- (Tett \& Bunnet, 2003) indica que la relación entre los rasgos de personalidad y la performance no es lineal, sino que está moderada por la importancia que la «activación» de ese rasgo pueda tener en la tarea que el trabajo en sí mismo implica, y la valoración que se le dé tanto a nivel personal, como organizacional y en la sociedad. Asimismo, algunos autores argumentan que, si se contextualizan, los rasgos de personalidad pueden ser mejores predictores de la satisfacción y el rendimiento laboral que si se los considera en forma global. Sería interesante el estudio, desde el modelo de rasgos positivos aquí tratado (PPM), de lo que algunos denominan personalidad laboral - work personality - entendida como patrones de conductas, pensamientos y emociones que se asocian al rol laboral (p. ej. Heller et al., 2009). Estos aspectos deberán ser tenidos en cuenta en futuros trabajos sobre la temática.

Además, futuras líneas de investigación deberán ampliar el abanico de variables, integrando el estudio de los rasgos positivos de personalidad analizados desde el PPM en otros grupos poblacionales y en relación con otras medidas de rendimiento (p. ej. rendimiento académico en estudiantes de nivel medio y/o universitario). 


\section{Referencias}

American Psychiatric Association (2013). Diagnostic andstatisticalmanual of mental disorders (5a ed.). Arlington, VA: American Psychiatric Publishing. https://doi.org/10.1176/appi.books.9780890425596 Anderson, G. \& Viswesvaran, C. (1998). An update of the validity of personality scales in personnel selection: A meta-analysis of studies published after 1992. Paper presented at the 13th Annual Conference of the Society for Industrial and Organizational Psychology, Dallas, TX.

Barrick, M.R. \& Mount, M.K. (1991). The Big Five personality dimensions and job performance: A meta-analysis. Personnel Psychology, 44(1), 1-26. http://dx.doi.org/10.1111/j.1744-6570. 1991.tb00688.x

Barrick, M. R., Mount, M. K. \& Judge, T. A. (2001). Personality and Performance at the Beginning of the New Millennium: What Do We Know and Where Do We Go Next? International Journal of Selection and Assessment, 9, 9-30. https://doi. org/10.1111/1468-2389.00160

Bowling, N.A. \& Burns, G. N. (2010). A comparison of work-specific and general personality measures as predictors of work and nonwork criteria. Personality and Individual Differences, 49, 95-101. https://doi.org/10.1016/j.paid.2010.03.009

Cameron, K.S., Brigth, D. \& Caza, A. (2004). Exploring the relationship between organizational virtuosness and performance. American Behavioral Scientist, 47, 1-24. http:// dx.doi.org/10.1177/0002764203260209

Costa, P.T. \& McCrae, R. R. (1985). The NEO Personality Inventory Manual. Odessa, FL: Psychological Assessment Resources. https://doi.org/10.1037/t07564-000

Christiansen, N. D. \& Tett, R. P. (2008). Toward a better understanding of the role of situations in linking personality, work behavior, and job performance. Industrial and Organizational Psychology, 1, 312-316. https://doi.org/10.1111/j.1754-9434.2008.00054.x 
De la Iglesia, G. \& Castro Solano, A. (2018). The Positive Personality Model (PPM): a new conceptual framework for personality assessment. Frontiers in Psychology, 9, 2025. https://doi. org/10.3389/fpsyg.2018.02027

DeNeve, M.K. \& Harris, C. (1998). The Happy Personality: A Meta-Analysis of 137 Personality Traits and Subjective WellBeing. Psychological Bulletin, 124, 197-229. https://doi.org/ 10.1037/0033-2909.124.2.197

Drozd, F., Mork, L., Nielsen, B., Raeder, S. \& Bjørkli, C. A. (2014). Better Days-A randomized controlled trial of an internetbased positive psychology intervention. The Journal of Positive Psychology, 9(5), 377-388. https://doi.org/10.1080/17439760.2 014.910822

Duan, W., Ho, S. M.Y., Yu, B., Tang, X., Zhang, Y., Li, T. \& Yuen, T. (2012). Factor structure of the Chinese virtues questionnaire. Research on Social Work Practice, 22(6), 680-688. https://doi. org/10.1177/1049731512450074

Dubreuil, P., Forest, J., Gillet, N., Fernet, C., Thibault-Landry, A., Crevier-Braud, L. \& Girouard, S. (2016). Facilitating wellbeing and performance through the development of strengths at work: Results from an intervention program. Journal of Applied Positive Psychology, 1(1-3), 1-19. https://doi.org/10.1007/ s41042-016-0001-8

Elston, F. \& Boniwell, I. (2011). A grounded theory study of the value derived by women in financial services through a coaching intervention to help them identify their strengths and practice using them in the workplace. International Coaching Psychology Review, 6(1), 16-32.

Gander, F., Proyer, R.T., Ruch, W. \& Wyss, T. (2012). Strength-based positive interventions: Further evidence for their potential in enhancing well-being. Journal of Happiness Studies, 14, 12411259. https://doi.org/10.1007/s10902-012-9380-0

Harzer C., Mubashar T. \& Dubreuil P. (2017). Character strengths and strength-related person-job fit as predictors of work- 
related wellbeing, job performance, and workplace deviance. Wirtschaftspsychologie, 19(3), 23-38.

Harzer, C. \& Ruch, W. (2014). The role of character strengths for task performance, job dedication, interpersonal facilitation, and organizational support. Human Performance. 27(3), 183-205. https://doi.org/10.1080/08959285.2014.913592

Heller, D., Ferris, D. L., Brown, D. \& Watson, D. (2009). The Influence of Work Personality on Job Satisfaction: Incremental Validity and Mediation Effects. Journal of Personality, 77(4), 1051-1084. https://doi.org/10.1111/j.1467-6494.2009.00574.x

Jugde, T.A., Heller, D. \& Mount, M.K. (2002). Five-Factor Model of Personality and Job Satisfaction: A Meta-Analysis. Journal of Applied Psychology, 87(3), 530-541. https://doi. org/10.1037/0021-9010.87.3.530

Keyes, C.L.M. (2002). The mental health continuum: From languishing to flourishing in life. Journal of Health and Social Behavior, 43, 207-222. https://doi.org/10.2307/3090197

Keyes, C. L. M. (2005). The subjective well-being of America's youth: toward a comprehensive assessment. Adolesc. Fam. Health, 4, 3-11.

Krueger, R.F., Derringer, J., Markon, K.E., Watson, D. \& Skodol, A. E. (2013). The Personality Inventory for DSM-5-Brief Form (PID-5-BF)_Adult. Massachusetts: American Psychiatric Association.

Lamers, S.M.A., Westerhof, G.J., Kovács, V. \& Bohlmeijer, E.T. (2012). Differential relationships in the association of the Big Five personality traits with positive mental health and psychopathology. Journal of Research in Personality, 46(5), $517-$ 524. https://doi.org/10.1016/j.jrp.2012.05.012

Lavy, S. \& Littman-Ovadia, H. (2016). My better self: Using strengths at work and work productivity, organizational citizenship behavior and satisfaction. Journal of Career Development, 1-15. https://doi.org/10.1177/0894845316634056 
Leary, T. (1957). Interpersonal diagnosis of personality. New York: Ronald Press.

Littman-Ovadia,H. \&Lavy, S. (2016). Going the extra mile: Perseverance as a key character strength at work. Journal of Career Assessment, 24(2), 240-252. https://doi.org/10.1177/1069072715580322

Littman-Ovadia, H., Lavy, S. \& Boiman-Meshita, M. (2016). When theory and research collide: Examining correlates of signature strengths use at work. Journal of Happiness Studies, 18(2), $527-$ 548. https://doi.org/10.1007/s10902-016-9739-8

Lupano Perugini, M.L., De la Iglesia, G., Castro Solano, A. \& Keyes, C.L. M. (2017). The Mental Health Continuum-Short Form (MHC-SF) in the Argentinean context: confirmatory factor analysis and measurement invariance. Europe's Journal of Psychology, 13, 93-108. https://doi.org/10.5964/ejop.v13i1.1163 Meyers, M. C. \& Van Woerkom, M. (2014). Effects of a Strengths Intervention on Work Engagement and Burnout: Positive Affect as a Mediator. Academy of Management Proceedings, 2014(1), 15558-15563. https://doi.org/10.5465/AMBPP.2014.15558 Millon, T. (1996). Disorders of Personality. New York: Wiley \& Sons. Millon, T. \& Everly, G. (1994). La personalidad y sus trastornos. Barcelona: Martícez Roca.

Mongrain, M. \& Anselmo-Matthews, T. (2012). Do positive psychology exercises work? A replication of Seligman et al. Journal of Clinical Psychology, 68(4), 382-389. https://doi.org/10.1002/jclp.21839 Moradi, S., Nima, A.A., Ricciardi, M.R., Archer, T. \& Garcia, D. (2014). Exercise, character strengths, well-being, and learning climate in the prediction of performance over a 6-month period at a call center. Frontiers in Psychology, 5, 497. https://doi.org/ 10.3389/fpsyg.2014.00497

Mount, M., Ilies, R. \& Johnson, E. (2006). Relationship of Personality Traits and Counterproductive Work Behaviors: The Mediating Effects of Job Satisfaction. Personnel Psychology, 59, 591-662. https://doi.org/10.1111/j.1744-6570.2006.00048.x 
Offer, D. \& Sabshin M. (1991). The diversity of normal behavior. New York: Basic Books.

Peterson, C. \& Seligman, M.E.P. (2004). Character strengths and virtues: A handbook and classification. Washington, DC: American Psychological Association; Oxford University Press.

Salgado, J.F. (1997). The five factor model of personality and job performance in the European Community. Journal of Applied Psychology, 82(1), 30-43. http://dx.doi.org/10.1037/0021-9010. 82.1 .30

Salgado, J.F. (2002). The Big Five Personality Dimensions and Counterproductive Behaviors. International Journal of Selection and Assessment, 10(1-2), 117-125. https://doi.org/10.1111/ 1468-2389.00198

Seligman, M. E. P. \& Csikszentmihalyi, M. (2014). Positive Psychology: An introduction. En M. Csikszentmihalyi (Ed.), Flow and the Foundations of Positive Psychology, 279-298. New York, NY: Springer US. https://doi.org/10.1007/978-94-017-9088-8_18

Strack, S. \& Lorr, M. (1994). Differentiating normal and abnormal personalities. New York, NY: Springer.

Strickhouser, J.E., Zell, E. \& Krizan, Z. (2017). Does personality predict health and well-being? A metasynthesis. Health Psychology, 36(8), 797-810. http://dx.doi.org/10.1037/hea0000475

Swider, B. W. \& Zimmerman, R. D. (2010). Born to burnout: A metaanalytic path model of personality, job burnout, and work outcomes. Journal of Vocational Behavior, 76, 487-506. https:// doi.org/10.1016/j.jvb.2010.01.003

Tabachnick, B. G. \& Fidell, L.S. (2013). Using Multivariate Statistics (6th Ed.). Boston, MA: Pearson.

Tett, R. P. \& Burnett, D. B. (2003). A personality trait based interactionist model of job performance. Journal of Applied Psychology, 88, 500-517. https://doi.org/10.1037/0021-9010.88.3.500

Tett, R. P., Jackson, D. N. \& Rothstein, M. (1991). Personality measures as predictors of job performance: A meta-analytic 
review. Personnel Psychology, 44(4), 703-742. http://dx.doi.org/ 10.1111/j.1744-6570.1991.tb00696.x

Wood, A. M., Linley, P.A., Matlby, J., Kashdan, T. B. \& Hurling, R. (2011). Using personal and psychological strengths leads to increases in well-being over time: A longitudinal study and the development of the strengths use questionnaire. Personality and Individual Differences, 50, 15-19. https://doi.org/10.1016/j. paid.2010.08.004

Young, K. C., Kashdan, T. B. \& Macatee, R. (2014). Strength balance and implicit strength measurement: New considerations for research on strengths of character. The Journal of Positive Psychology, 10(1), 17-24. https://doi.org/10.1080/17439760.2 014.920406

Zhai, Q., Willis, M., O'Shea, B., Zhai, Y. \& Yang, Y. (2012). Big Five personality traits, job satisfaction and subjective wellbeing in China. International Journal of Psychology, 48(6), 1099-1108. https://doi.org/10.1080/00207594.2012.732700

Zimmerman, R. (2008). Understanding the impact of personality traits on individuals turnover decisions: A meta-analytic path model. Personnel Psychology, 61(2), 309-348. https://doi.org/10. $1111 /$ j.1744-6570.2008.00115.x

Recibido: 5 de junio, 2018 Revisado: 1 de febrero, 2019 Aceptado: 4 de febrero, 2019 